Classification

Physics Abstracts

61.14Dc-61.14Di

\title{
Energy-filtered HREM images of valence-loss electrons
}

\author{
Zhong Lin Wang $\left({ }^{1,2}\right)$ and James Bentley $\left({ }^{1}\right)$
}

( $\left.{ }^{1}\right)$ Metals and Ceramics Division, Oak Ridge National Laboratory, P.O. Box 2008, Oak Ridge, TN, 37831-6376, U.S.A.

( $\left.{ }^{2}\right)$ Department of Materials Science and Engineering, The University of Tennessee, Knoxville, TN, 37996-2200, U.S.A.

(Received June 05, 1991; accepted January 15, 1992)

\begin{abstract}
A theory is proposed to include the effects of valence excitations in electron image simulations for high-resolution electron microscopy (HREM) based on the single inelastic scattering model. Under the small thickness approximation, this general theory reduces to the simplified theory of perfectly delocalized inelastic scattering model, in which the image can be considered to be an incoherent sum of those incident electrons of different energies weighted by the intensity distribution in the electron energy-loss spectrum from the area where the pattern was taken. The main effect of valence-loss is to introduce a focus shift due to chromatic aberration, resulting in contrast variation (or reversal) of the image. The generalization of this theory for simulations of interface images with considering surface and interface plasmon excitations is given. Calculations for GaAs surface profile images are demonstrated to show the effect of inelastic localization.
\end{abstract}

\section{Introduction.}

Electron inelastic scattering is usually classified into three different processes. Plasmon (or valence) excitation, which characterizes the transitions of electrons from the valence band to the conduction band, involves an energy-loss in the range of 5-30 eV and an angular spreading of less than $0.2 \mathrm{mrad}$ for high energy electrons. This scattering process is the main inelastic even in highresolution electron microscopy (HREM) imaging, because the inelastic mean-free-path (MFP) for valence excitation is quite short compared to those for the following two processes. Atomic core-shell excitations introduce an energy-loss in the range of a few hundreds to thousands $\mathrm{eV}$ with an angular spreading of an order $\vartheta_{\mathrm{E}}=\frac{\Delta E}{2 E_{0}}$, where $\Delta E$ is the electron energy-loss and $E_{0}$ is the incident electron energy. Since the probability of exciting the core shells is very small, this process is usually neglected in HREM image simulations. Thermal diffuse scattering (TDS) or phonon scattering does not introduce any significant energy-loss but produces large momentum transfer, which can scatter the electrons out of the selecting angular range of the objective aperture. This is partly equivalent to introducing an absorption factor in elastic HREM image simulation. Therefore, valence excitation and phonon scattering are the inelastic scattering processes one needs to consider in HREM image simulations. 
In high-energy electron diffraction and imaging, valence excitations are delocalized inelastic scattering processes of many excited states of different energies and momentum transfers. The incoherence of the electrons in these excited states makes the theoretical treatment extremely difficult because the electrons that have excited a specific state have to be considered as a single stream propagating through the rest of the crystal. The diffraction pattern is formed by the incoherent sum of these different streams at the exit face of the crystal. In HREM, valence excitations contribute significant details to the images and become more important if the crystal is thicker [13]. Theoretical calculations by these authors are based on a model in which the valence excitation is considered occurring at the entrance face of the crystal slab, the final image being an incoherent superposition of all the electrons with different incident energies. This model was introduced by assuming that the valence excitation is a perfectly delocalized inelastic scattering process, so that whether the inelastic events occur inside or outside the crystal, the image calculations are not affected. This model, in principle, is valid for very thin crystals. However, the questions remain as to how thin the crystal should be to ensure the validity of the model, and what the wave mechanics basis is of this simplified picture. In addition, the excitations of surface and interface plasmons have not been included in these calculations. In order to address these questions, one needs to start from the inelastic scattering theory of high-energy electrons.

The multislice approach is widely applied for calculating images formed with elastically scattered electrons [4]. The application of this multislice method for solving the coupled Schrodinger equations [5] gave birth to a generalized multislice theory of elastic and inelastic electron scattering. The generalized multislice theory considers the electrons in different excited states as a group of waves, the propagation of which is governed by a matrix formula [6]. In practice, this matrix formula can be simplified for the cases of a single excited state, such as single electron core-shell excitations and thermal diffuse scattering (TDS). However, the computation becomes huge if the number of excited states is large, as with valence excitations. In this case, the calculations become unfeasible in practice.

To reduce the computation time, we have recently introduced a new dynamical theory for describing inelastic electron scattering both in perfect crystals and in crystals with defects, and it has been applied to localized inelastic scattering [7]. The phase correlations of the localized inelastic scattering occurring at different atomic sites can be statistically evaluated before any numerical calcultations. The application of this theory for calculating the HREM images formed by phonon scattered electrons has been described in our earlier paper. In this paper, this new theory is applied to treat the delocalized valence excitations. A method is introduced to include the effects of valence excitations in the simulation of electron diffraction patterns and images in HREM. The wave mechanics basis of this method is illustrated and its relationship with the simplified model proposed by other authors [1-3] is illustrated. Finally, the newly derived theory is generalized for simulating HREM images of surfaces and interfaces with due consideration of surface and interface plasmon excitations, and some corresponding calculations are given.

\section{Basic theory.}

Considering the incident electron and the crystal as a whole system, if $\Psi_{0}$ describes the elastically scattered wave of energy $E_{0}=E$, and $\Psi_{n}$ describes the inelastically scattered wave of energy $E_{n}=E-\varepsilon_{n}$, with $n=1,2 \ldots$, under the single inelastic scattering approximation, the electron scattering with the presence of the $n^{\text {th }}$ excited state is determined by [5],

$$
\left(\nabla^{2}+k_{n}^{2}+\frac{2 m_{0} e}{\hbar^{2}} V(\mathbf{r})\right) \Psi_{n} \approx \frac{2 m_{0}}{\hbar^{2}} H_{n 0}^{\prime}(\mathbf{r}) \Psi_{0}, \text { with } n=0,1,2 \ldots
$$


where

$$
k_{n}^{2} \equiv \frac{2 m_{0}}{\hbar^{2}} E_{n}
$$

$V$ is the crystal potential, and $H_{n 0}^{\prime}$ is the transition matrix of the corresponding inelastic scattering process. The solution of equation (1a) can be written in the form [8],

$$
\Psi_{n} \equiv \phi_{n}(\mathbf{r}) \Psi_{n}^{0}(\mathbf{r})
$$

where $\Psi_{n}^{0}(\mathbf{r})$ satisfies the elastic scattering Schrodinger equation,

$$
\left(\nabla^{2}+k_{n}^{2}\right) \Psi_{n}^{0}=-\frac{2 m_{0} e}{\hbar^{2}} V(\mathbf{r}) \Psi_{n}^{0}
$$

Equation (2) means that the effects of inelastic scattering can be considered as a positional modulation to the elastically scattered wave. It is important to note that the modulation function $\phi_{n}(\mathbf{r})$ depends not only on $z$ but also on $(x, y) . \Psi_{n}^{0}(\mathbf{r})$ is the full multislice solution of equation (3) with considering the boundary conditions. By neglecting the $\nabla^{2} \phi_{n}$ term under the small-angle approximation, for $k_{x, y}<<k_{z}$, so that the $\partial \phi_{n}(\mathbf{r}) / \partial x$ and $\partial \phi_{n}(\mathbf{r}) / \partial y$ terms can be neglected, and using equation (3), equation (1a) becomes

$$
\frac{\partial \phi_{n}(\mathbf{r})}{\partial z} \approx \alpha H_{n 0}^{\prime}(\mathbf{r}) \Psi_{0}^{0}(\mathbf{r})\left[\frac{\partial \Psi_{n}^{0}(\mathbf{r})}{\partial z}\right]^{-1} \phi_{0}(\mathbf{r})
$$

and $\alpha=m_{0} / \hbar^{2}$. Numerical calculations have shown that the $\frac{\partial \Psi_{n}^{0}(\mathbf{r})}{\partial z}$ function never goes to zero.

Assuming that the crystal is so thin that the absorption effects are negligible, i.e., $\phi_{0}(\mathbf{r}) \approx 1$, and considering the boundary condition $\phi_{n}(b, 0)=0$, the solution of equation (4) is,

$$
\phi_{n}(\mathbf{b}, z) \approx \alpha \int_{0}^{z} \mathrm{~d} z^{\prime} H_{n 0}^{\prime}\left(\mathbf{b}, z^{\prime}\right) \Psi_{0}^{0}\left(\mathbf{b}, z^{\prime}\right)\left[\frac{\partial \Psi_{n}^{0}\left(\mathbf{b}, z^{\prime}\right)}{\partial z}\right]^{-1}
$$

or

$$
\Psi_{n}(\mathbf{b}, z) \approx \alpha \int_{0}^{z} \mathrm{~d} z^{\prime}\left\{H_{n 0}^{\prime}\left(\mathbf{b}, z^{\prime}\right) S_{n 0}\left(\mathbf{b}, z^{\prime}\right)\right\} \Psi_{n}^{0}(\mathbf{b}, z)
$$

where

$$
S_{n 0}(\mathbf{b}, z) \equiv \Psi_{0}^{0}(\mathbf{b}, z) / \frac{\partial \Psi_{n}^{0}(\mathbf{b}, z)}{\partial z}, \text { and } \mathbf{b}=(x, y) .
$$

This is the solution of single inelastic scattering under the first order approximation, which has been applied to treat the localized inelastic processes of single electron excitation and thermal diffuse scattering $[7,9]$. In the theory of inelastic electron scattering, the most difficult part is to perform the incoherent sum of the intensities contributed by the electrons after exciting different crystal states, because there are too many crystal states to be handled with the limited computation power. With equation (5), we are able to perform this incoherent sum analytically before any numerical calculations. The $S_{n m}(\mathbf{r})$ function appeared in the integration operation is responsible for forming the Kikuchi pattern.

Equation (5b) appears in a different form compared to either the Bloch wave theory or the multislice theory. It has been proved that equation (5) is equivalent to the inelastic multislice theory (see Appendix A). This firmly establishes the relationship of this new theory with other 
existing theories, and confirms the validities of the approximations based on which equations (5) was derived.

In the multislice theory, a crystal is cut into many slices in the $z$ direction and the atomic structure in each slice is projected on a plane perpendicular to the $z$ axis. Thus it is not straightforward to find $\frac{\partial \Psi_{n}^{0}(\mathbf{r})}{\partial z}$ by differentiating $\Psi_{n}^{0}$ with respect to $z$. For this reason one starts from equation (3), where by taking $\Psi_{n}^{0}(\mathbf{b}, z)=\Phi_{n}^{0}(\mathbf{b}, z) \exp \left(i \mathbf{k}_{n} \cdot \mathbf{r}\right)$ and neglecting the $\frac{\partial^{2} \Phi_{n}^{0}}{\partial z^{2}}$ term under the small angle approximation, equation (3) becomes

$$
\frac{\partial \Phi_{n}^{0}(\mathbf{r})}{\partial z}=-\left\{\frac{2 m_{0} e}{\hbar^{2}} V(\mathbf{r}) \Phi_{n}^{0}+\left(\frac{\partial^{2}}{\partial x^{2}}+\frac{\partial^{2}}{\partial y^{2}}\right) \Phi_{n}^{0}+i 2\left(k_{n x} \frac{\partial}{\partial x}+k_{n y} \frac{\partial}{\partial y}\right) \Phi_{n}^{0}\right\} / i 2 k_{n z}
$$

Then equation (5c) becomes

$$
S_{n m}(\mathbf{r})=\frac{\Phi_{m}^{0} \exp \left(i\left(\mathbf{k}_{m}-\mathbf{k}_{n}\right) \cdot \mathbf{r}\right)}{i k_{n z} \Phi_{n}^{0}-\left\{\frac{2 m_{0} e}{\hbar^{2}} V(\mathbf{r}) \Phi_{n}^{0}+\left(\frac{\partial^{2}}{\partial x^{2}}+\frac{\partial^{2}}{\partial y^{2}}\right) \Phi_{n}^{0}+i 2\left(k_{n x} \frac{\partial}{\partial x}+k_{n y} \frac{\partial}{\partial y}\right) \Phi_{n}^{0}\right\} / i 2 k_{n z}}
$$

In the following discussions, $\Phi_{n}^{0}$ is the solution of the elastic scattering equation (3), which can be solved using the multislice method for high-energy electrons $[4,10]$. In the right-hand side of equation (7), the first term in the denominator is much larger than the remaining terms, especially for valence excitations, so that

$$
S_{n m}(\mathbf{r}) \approx \frac{\Phi_{m}^{0}(\mathbf{r}) \exp \left(-i \mathbf{q}_{m n} \cdot \mathbf{r}\right)}{i k_{n z} \Phi_{n}^{0}(\mathbf{r})}
$$

with

$$
\mathbf{q}_{m n} \equiv \mathbf{k}_{n}-\mathbf{k}_{m}
$$

being the momentum transfer during the inelastic scattering from the $m^{\text {th }}$ state to the $n^{\text {th }}$ state. The $z$ component of the momentum transfer $q_{n 0}$ is only related to the electron energy loss by

$$
\left(q_{n 0}\right)_{z}=q_{n} \approx \frac{k_{0} \Delta E}{2 \gamma E_{0}}
$$

where $\Delta E=\hbar \omega$ is the electron energy-loss and $\gamma=\left(1-v^{2} / c^{2}\right)^{-1 / 2}$. For the convenience of discussion in the next section, one first introduces the concept of the density operator in inelastic scattering. In general, for $n \neq m$, the interaction of an external electron with a crystal can be stated as

$$
\begin{aligned}
H_{n m}^{\prime}(\mathbf{r}) & =\left\langle m\left|\frac{1}{4 \pi \varepsilon_{0}}\left\{\sum_{j} \frac{e^{2}}{\left|\mathbf{r}-\mathbf{r}_{j}\right|}-\sum_{k} \frac{Z_{k} e^{2}}{\left|\mathbf{r}-\mathbf{R}_{k}\right|}\right\}\right| n\right\rangle \\
& =\frac{e^{2}}{8 \pi^{3} \varepsilon_{0}} \int \frac{\mathbf{d g}}{\mathbf{g}^{2}} \exp (i \mathbf{g} \cdot \mathbf{r}) \rho_{n m}(\mathbf{g}),
\end{aligned}
$$

where $\mathbf{r}_{j}$ and $\mathbf{R}_{j}$ are the positions of the $j^{\text {th }}$ crystal electron and the $k^{\text {th }}$ nucleus respectively, and

$$
\rho_{n m}(\mathbf{g}) \equiv\left\langle m\left|\sum_{j} \exp \left(-i \mathrm{~g} \cdot \mathbf{r}_{j}\right)\right| n\right\rangle
$$


$\rho_{n m}(\mathrm{~g})$ is the Fourier-transformed density operator in the Heisenberg representation and is usually a complex function associated directly with the electron distribution inside the crystal. In valence excitations, the density operator can be correlated with the dielectric response function of the crystal.

\section{Diffraction of valence-loss electrons.}

Before considering the images formed by inelastically scattered electrons, one first calculates the diffraction pattern formed by the electrons after valence excitations. This section is described for the convenience of image calculation in the next section, because the effect of the objective lens need not be considered in diffraction pattern calculation. For easy discussion, one assumes that $H_{n m}^{\prime}$ does not depend on $z$ for volume plasmon excitations. The dependence of $H_{n m}^{\prime}$ on $z$ for surface plasmon excitations will be discussed in sections 6 and 7. At the crystal exit face $z=d$, equation ( $5 b)$ can be rewritten as,

$$
\Psi_{n}(\mathbf{b}, d)=\alpha H_{n 0}^{\prime}(\mathbf{b}) Y(\mathbf{b}, \omega)
$$

where

$$
Y(\mathbf{b}, \omega)=\int_{0}^{d} \mathrm{~d} z \Psi_{0}^{0}(\mathbf{b}, z) / \frac{\partial \Psi_{0}^{0}(\mathbf{b}, z, \omega)}{\partial z} \Psi_{0}^{0}(\mathbf{b}, d, \omega)
$$

For easier notation, $\Psi_{n}^{0}(\mathbf{b}, d)$ is replaced by $\Psi_{0}^{0}(\mathbf{b}, d, \omega)$, where $\hbar \omega$ is the electron energy loss. The change of symbol simply neglects the $q$ dependence of $\Psi_{n}^{0}$ (i.e., presuppose that all states are non-degenerate). For small angle plasmon excitation, the introduction of a small angle spreading (less than $0.2 \mathrm{mrad}$ ) actually does not affect the final calculated results. Equation (12) is the fundamental equation used for following calculations. Now one considers the energy-filtered diffraction patterns formed by the valence excited electrons. Taking the Fourier transform of equation (12a), the contributions of all the valence excited states to the final electron diffraction pattern are added incoherently, in reciprocal space $\mathrm{g}_{\mathrm{b}}=\left(\mathrm{g}_{x}, \mathrm{~g}_{y}\right)$,

$$
I_{\mathrm{v}}\left(\mathrm{g}_{\mathrm{b}}\right)=\sum_{n}\left|\Psi_{n}\left(\mathrm{~g}_{\mathrm{b}}, d\right)\right|^{2}=\left[\frac{\alpha d}{8 \pi^{3} \varepsilon_{0}}\right]^{2} \sum_{n} \mid H_{n 0}^{\prime}\left(\mathrm{g}_{\mathrm{b}}\right) \otimes Y\left(\mathrm{~g}_{\mathrm{b}},\left.\omega\right|^{2},\right.
$$

where the sum of $n$ is over all the possible valence states of different energies and different momenta; $\otimes$ indicates a convolution operation of $\mathbf{g}_{b} ; Y\left(\mathbf{g}_{b}, \omega\right)$ is the Fourier transform of the function defined in equation (12b). By using equation (10) and expanding the convolution operation, equation (13) can be rewritten as

$$
I_{\mathbf{v}}\left(\mathbf{g}_{\mathbf{b}}\right)=C_{0} \int \mathbf{d} \tau_{\mathbf{b}} \int \mathbf{d} \tau_{\mathbf{b}}^{\prime} \frac{Y\left(\mathbf{g}_{\mathbf{b}}-\tau_{\mathbf{b}}, \omega\right) Y^{*}\left(\mathbf{g}_{\mathbf{b}}-\tau_{\mathbf{b}}^{\prime}, \omega\right)}{\tau^{2} \tau^{\prime 2}} S\left(\tau, \tau^{\prime}\right)
$$

where $C_{0}=\frac{\alpha^{2} e^{4}}{\varepsilon_{0}^{2}} ; \tau^{2}=\tau_{\mathrm{b}}^{2}+\tau_{z}^{2}$ with $\tau_{z}=q_{n}=\frac{k_{0} \hbar \omega}{2 \gamma E_{0}} ;$ and $S$ is defined as the mixed dynamic form factor,

$$
S\left(\mathbf{g}, \mathbf{g}^{\prime}\right) \equiv \sum_{n} \rho_{0 n}(\mathbf{g}) \rho_{n 0}\left(-\mathbf{g}^{\prime}\right)
$$

For electron collective excitations, the mixed dynamic form factor is related to the generalized dielectric function by $[13,14]$

$$
S\left(\mathbf{g}, \mathbf{g}^{\prime}\right)=\int_{-\infty}^{\infty} \mathrm{d} \omega S\left(\mathbf{g}, \mathbf{g}^{\prime}, \omega\right)
$$


and

$$
S\left(\mathbf{g}, \mathbf{g}^{\prime}, \omega\right)=\frac{i \hbar \varepsilon_{0} V}{2 \pi e^{2}\left[1-\mathrm{e}^{-\beta \hbar \omega}\right]}\left\{\frac{\mathbf{g}^{2}}{\varepsilon_{\mathbf{g g}^{\prime}}(\omega)}-\frac{\mathbf{g}^{\prime 2}}{\varepsilon_{\mathbf{g}^{\prime} \mathbf{g}}^{*}(\omega)}\right\}
$$

where $\beta=\frac{1}{k_{\mathrm{B}} T^{\prime}} T$ is the temperature of the system and $V$ is the volume of the crystal. Putting equation (17) into (14),

$$
\begin{aligned}
I_{\mathbf{v}}\left(\mathbf{g}_{\mathbf{b}}\right)= & C_{0} \int \mathrm{d} \tau_{\mathbf{b}} \int \mathrm{d} \tau_{\mathbf{b}}^{\prime} Y\left(\mathbf{g}_{\mathbf{b}}-\tau_{\mathbf{b}}, \omega\right) Y^{*}\left(\mathrm{~g}_{\mathbf{b}}-\tau_{\mathbf{b}}^{\prime}, \omega\right) \\
& \times \int_{-\infty}^{\infty} \mathrm{d} \omega^{\prime} \frac{i \hbar \varepsilon_{0} V}{2 \pi e^{2}\left[1-\mathrm{e}^{\left.-\beta \hbar \omega^{\prime}\right]}\right.}\left\{\frac{1}{\tau^{\prime 2} \varepsilon_{\tau \tau^{\prime}}\left(\omega^{\prime}\right)}-\frac{1}{\tau^{2} \varepsilon^{*}{ }_{\tau^{\prime} \tau}\left(\omega^{\prime}\right)}\right\}
\end{aligned}
$$

Assuming that the distribution of incident electrons inside the crystal is not significantly affected by a small energy-loss, so that $Y\left(g_{b}-\tau_{b}, \omega\right)$ can be approximately included in the integration of $\omega^{\prime}$ (In numerical calculations, we found this is actually an excellent approximation), and considering condition $\left|\hbar \omega / k_{\mathrm{B}} T\right|>>1$ for plasmon excitations, equation (18) becomes

$$
I_{\mathbf{v}}\left(\mathbf{g}_{\mathrm{b}}\right) \approx C_{1} V \int \mathrm{d} \tau \int \mathrm{d} \tau^{\prime} \int_{0}^{\infty} \mathrm{d} \omega \frac{1}{\tau^{\prime 2}} \operatorname{Im}\left[-\frac{1}{\varepsilon_{\tau \tau^{\prime}}(\omega)} Y\left(\mathbf{g}_{\mathrm{b}}-\tau_{\mathrm{b}}, \omega\right) Y^{*}\left(\mathrm{~g}_{\mathrm{b}}-\tau_{\mathrm{b}}^{\prime}, \omega\right)\right]
$$

where $C_{1}=\frac{e^{2} m_{0}^{2}}{\pi \varepsilon_{0} \hbar^{3}}$. Equation (19) is a generalized intensity distribution in the valence-loss electron diffraction pattern. Now consider a case where the dielectric function depends only on the electron energy-loss, for a homogeneous medium

$$
\frac{1}{\varepsilon_{\tau \tau^{\prime}}(\omega)}=\frac{1}{\varepsilon(\omega)} \delta\left(\tau-\tau^{\prime}\right) \delta\left(\tau_{z}-q_{n}\right) / V
$$

Thus equation (19) becomes

$$
I_{\mathrm{v}}\left(\mathrm{g}_{\mathrm{b}}\right)=4 \pi^{2} k_{0}^{2} \int_{0}^{\infty} \mathrm{d} \omega \int \mathrm{d} \tau_{\mathrm{b}} \frac{\mathrm{d}^{2} \sigma\left(\tau_{\mathrm{b}}, \omega\right)}{\mathrm{d} \omega \mathrm{d} \tau_{\mathrm{b}}}\left|Y\left(\mathrm{~g}_{\mathrm{b}}-\tau_{\mathrm{b}}, \omega\right)\right|^{2}
$$

where the electron double-differential scattering cross-section function is defined [15] as

$$
\frac{\mathrm{d}^{2} \sigma\left(\tau_{\mathrm{b}}, \omega\right)}{\mathrm{d} \omega \mathrm{d} \tau_{\mathrm{b}}}=\frac{e^{2}}{4 \pi^{3} \varepsilon_{0} v^{2}} \frac{1}{\tau_{\mathrm{b}}^{2}+q_{n}^{2}} \operatorname{Im}\left[-\frac{1}{\varepsilon(\omega)}\right]
$$

Therefore, the diffraction pattern is composed of the incoherent addition of all the electrons with different energy-losses $\hbar \omega$ and momentum transfers, weighted by the double-differential scattering cross-section. Equation (22) is the basic equation for calculating inelastic diffraction patterns formed by valence excited electrons. 


\section{Energy-filtered HREM images of valence-loss electrons.}

For image simulations, considering first the case of a perfect objective lens (i.e. no spherical and chromatic aberration) without objective aperture. Adding the images formed by the electrons after exciting different valence states incoherently, following the analogous procedures as for diffraction case, from equation (12a) we have,

$$
\begin{aligned}
& I_{\mathbf{v}}(\mathbf{b})=(2 \pi)^{6} \sum_{n}\left|\Psi_{n}(\mathbf{b}, d)\right|^{2} \\
& \approx C_{0} \int_{-\infty}^{\infty} \mathrm{d} \omega \int \mathrm{dq} \int \mathrm{dq}^{\prime} \frac{\hbar \varepsilon_{0} V}{\pi e^{2}\left[1-\mathrm{e}^{-\beta \hbar \omega}\right]} \operatorname{Im}\left\{-\frac{\exp \left(i\left(\mathbf{q}-\mathbf{q}^{\prime}\right) \cdot \mathbf{b}\right.}{q^{\prime 2} \varepsilon_{\mathbf{q q}}(\omega)}\right\}\left|Y\left(\mathbf{b}, E_{0}-\hbar \omega\right)\right|^{2} .
\end{aligned}
$$

This equation represents the image formed by the valence-loss electrons. In practice, a nonperfect objective lens has different contrast transfer functions for electrons with different energylosses, therefore equation (23) can be generalized as

$$
\begin{aligned}
I_{\mathbf{v}}(\mathbf{b}) & \approx C_{0} \int_{-\infty}^{\infty} \mathrm{d} \omega \int \mathrm{dq} \int \mathrm{d} \mathbf{q}^{\prime} \frac{\hbar \varepsilon_{0} V}{\pi e^{2}\left[1-\mathbf{e}^{-\beta \hbar \omega}\right]} \operatorname{Im}\left\{-\frac{\exp \left(i\left(\mathbf{q}-\mathbf{q}^{\prime}\right) \cdot \mathbf{b}\right.}{q^{\prime 2} \varepsilon_{\mathbf{q q}^{\prime}}(\omega)}\right\} \times \\
& \times\left|Y\left(\mathbf{b}, E_{0}-\hbar \omega\right) \otimes F_{\mathrm{OB}}(\mathbf{b}, \omega)\right|^{2},
\end{aligned}
$$

where $F_{\mathrm{OB}}$ represents the effect of the objective lens on electrons of energy $E_{0}-\hbar \omega$, the Fourier transform of which is defined in reciprocal space as

$$
F_{\mathrm{OB}}(\tau, \omega)=B(\tau) \exp (i W(\tau))
$$

with

$$
W(\tau)=\lambda \tau^{2}\left[C_{\mathrm{s}} \lambda^{2} \tau^{2} / 8 \pi^{2}-\Delta f+\Delta f_{\mathrm{c}}\right] / 4 \pi,
$$

where $C_{\mathrm{s}}$ is the lens spherical aberration coefficient, $B$ defines the shape of the objective aperture, $\lambda$ is the energy-loss dependent electron wavelength, $\Delta f$ is the lens defocus, and $\Delta f_{\mathrm{c}}$ is the focus shift introduced by chromatic aberration

$$
\Delta f_{\mathrm{c}}=\frac{C_{\mathrm{c}} \Delta E}{E_{0}}
$$

where $C_{\mathrm{c}}$ is the chromatic aberration coefficient of the objective lens. For $C_{\mathrm{c}}=1.2 \mathrm{~mm}, \Delta E=$ $15 \mathrm{eV}$ and $E_{0}=200 \mathrm{keV}$, equation (26) yields $\Delta f_{\mathrm{c}}=90 \mathrm{~nm}$. This is a relatively large change and may produce contrast reversal in the image. Using equations (20) and (22), following procedures analogous to those used for deriving equation (21), equation (24) can be simplified for a homogeneous medium as

$$
I_{\mathrm{v}}(\mathbf{b}) \approx 4 \pi^{2} k_{0}^{2} \int_{0}^{\infty} \mathrm{d} \omega \frac{\mathrm{d} I(\omega)}{\mathrm{d} \hbar \omega}\left|Y(\mathbf{b}, \omega) \otimes F_{\mathrm{OB}}(\mathbf{b}, \omega)\right|^{2} .
$$

where the electron energy-loss function is defined as

$$
\frac{\mathrm{d} I(\omega)}{\mathrm{d} \omega}=\int_{0}^{\tau_{\mathrm{c}}} \mathrm{d} \tau_{\mathrm{b}} 2 \pi \tau_{\mathrm{b}} \frac{\mathrm{d}^{2} \sigma}{\mathrm{d} \omega \mathrm{d} \tau_{\mathrm{b}}}=\frac{e^{2}}{4 \pi^{2} \varepsilon_{0} \hbar v^{2}} \ln \left[1+\left(\frac{2 q_{\mathrm{c}} \gamma E_{0}}{k_{0} \hbar \omega}\right)^{2}\right] \operatorname{Im}\left[-\frac{1}{\varepsilon(\omega)}\right]
$$


$q_{c}$ is the cut-off of momentum transfer in valence excitation $[15] ; Y\left(g_{b}, \omega\right)$ is the Fourier transform of the $Y$ function defined by

$$
Y(\mathbf{b}, E)=\int_{0}^{d} \mathrm{~d} z \Psi_{0}^{0}\left(\mathbf{b}, z, E_{0}\right) / \frac{\partial \Psi_{0}^{0}(\mathbf{b}, z, E)}{\partial z} \Psi_{0}^{0}(\mathbf{b}, d, E),
$$

$\Psi_{0}^{0}\left(\mathbf{b}, z, E_{0}\right)$ and $\Psi_{0}^{0}(\mathbf{b}, z, E)$ are the multislice solutions of equaton (3) for electron beams of energy $E_{0}$ and $E=E_{0}-\hbar \omega$, respectively. $Y(\mathbf{b}, \omega)$ term contains the perturbation of the electron wave function by the energy-losses, the chromatic aberration or focus shift effect of the lens being introduced in $F_{\mathrm{OB}}(\mathbf{b}, \omega)$.

The inelastic wave of particular state excited at crystal depth $z$ is denoted by a location "tag", $S_{10}=\Psi_{0}^{0}\left(\mathbf{b}, z, E_{0}\right) / \frac{\partial \Psi_{0}^{0}(\mathbf{b}, z, E)}{\partial z}$. This function is responsible for the re-elastic scattering of the electrons after inelastic excitation and is the source for forming Kikuchi patterns. For very small energy losses, i.e., $\omega \rightarrow 0$, from equation (8a) one has $S_{10} \approx 1 / i k_{z}$. Thus the $S_{10}$ modulation becomes unimportant. For energy-losses larger than a few eV, the deviation of $S_{10}$ function becomes more and more significant with the increase of the crystal thickness $d$. In this case, the valence excitation cannot be considered as occurring at the entrance face of the crystal. All these effects are comprehensively included in equation (27). In general, for $\hbar \omega<<E_{0}$, it is possible to calculate $\Psi_{0}^{0}\left(\mathbf{b}, z, E_{0}-\hbar \omega\right)$ from $\Psi_{0}^{0}\left(\mathbf{b}, z, E_{0}\right)$ using the perturbation theory (see Appendix B).

It is important to point out that equations (21) and (27) were derived based on the single inelastic scattering model. In practice, the plural plasmon excitation obeys the Poission law, hence the relative intensity of the $m^{\text {th }}$ plasmon loss to the pure elastic peaks is $\frac{(d / \Lambda)^{m}}{m !}$, where $\Lambda$ is the MFP of plasmon excitation and is typically about $200-300 \mathrm{~nm}$ for $200 \mathrm{keV}$ electrons (see the calculated results in Sect. 6 and 7). In the crystal thickness range of about $50 \mathrm{~nm}$, the condition $(d / \Lambda)^{2}<<1$ is always satisfied. Therefore, in the thickness range for HREM imaging, the high order plasmon excitations can be neglected. In other words, the single plasmon scattering model is a good approximation. Therefore, equation (27) is a general formula for simulating HREM images of valence-loss electrons.

\section{Approaching the perfect delocalization model.}

To see the physical meaning of equations (21) and (27) more clearly, one considers a case in which the crystal thickness is assumed to be small. For delocalized valence excitations with energy-loss less than about $30 \mathrm{eV}$ and angular spreading less than $0.5 \mathrm{mrad}$, their perturbation to the wave function of the high-energy electrons may be neglected if the specimen is thin, i.e., $\Phi_{n}^{0} \approx \Phi_{0}^{0}$ (For specimens thinner than $20 \mathrm{~nm}$, this actually is an excellent approximation). From equation (8a), one approximately has

$$
S_{10}(\mathbf{r}) \approx \frac{\exp \left(-i q_{n} z\right)}{i k_{0}}
$$

so that

$$
Y(\mathbf{b}, E)=\frac{\exp \left(-i q_{n} d\right)-1}{k_{0} q_{n}} \Psi_{0}^{0}(\mathbf{b}, d, E) .
$$

For $q_{n} d<0.6$ (here 0.6 is chosen based on $\cos \left(q_{n} d\right) \approx 1$ and $\sin \left(q_{n} d\right) \approx q_{n} d$; this is a rough estimation for thickness), $Y(b, \omega) \approx \frac{d}{i k_{0}} \Psi_{0}^{0}(\mathbf{b}, d, \omega)$. Thus equation (21) becomes

$$
I_{\mathrm{v}}\left(\mathrm{g}_{\mathrm{b}}\right) \approx d^{2} \int_{0}^{\infty} \mathrm{d} \omega \frac{\mathrm{d} I(\omega)}{\mathrm{d} \hbar \omega} I_{\mathrm{els}}\left(\mathrm{g}_{\mathrm{b}}, E_{0}-\hbar \omega\right)
$$


where $I_{\mathrm{els}}\left(\mathrm{g}_{\mathrm{b}}, E\right)$ is the diffraction pattern formed by elastic electrons of incident energy $E$. Equation (32) means that the diffraction pattern formed by the valence excited electrons is an incoherent superposition of those elastic patterns formed by the electrons with different incident energies, weighted by the electron energy-loss function. For HREM images, equation (27) becomes

$$
I_{\mathrm{v}}(\mathbf{b})=d^{2} \int_{0}^{\infty} \mathrm{d} \omega \frac{\mathrm{d} I(\omega)}{\mathrm{d} \hbar \omega} I_{\mathrm{els}}\left(\mathbf{b}, E_{0}-\hbar \omega\right),
$$

where $I_{\mathrm{els}}(\mathbf{b}, E)$ is the image formed by the elastically scattering electrons of incidence energy $E$. Therefore, the inelastic image is formed by the incoherent addition of the electrons with different incident energies $E$, weighted by the intensity distribution in the EELS spectrum from the imaged area of the specimen.

Physically, equation (33) indicates that the non-localized valence excitations can be considered as occurring at the entrance face of the crystal for thin specimens. This agrees with the early treatment introduced by Gjønnes and Høier [16]. The final image contrast of the electrons after valence excitation is determined by the inelastic incident wave after penetrating the crystal, i.e. $\Psi_{0}^{0}(\mathbf{b}, z, E)$ at the exit face $z=d$. It is not necessary to consider the detailed excitation processes of the valence electrons. This is the result of the perfectly delocalized inelastic scattering model.

Under the small thickness approximation, $q_{n} d<0.6$, equation (33) establishes the wave mechanics basis of the model used by several authors [1-3]. The condition $q_{n} d=\frac{\pi \Delta E}{\gamma \lambda E_{0}} d<0.6$ sets a rough criterion for identifying the applicability of the simplified theory (Eq. (33)). For an energy loss of $10 \mathrm{eV}$ and $E_{0}=200 \mathrm{keV}$, yields $d<9 \mathrm{~nm}$. This value is approximately the localization of plasmon-losses [17].

\section{Effect of surface plasmon excitation.}

For simplification, the dependence of the interaction Hamiltonian $H^{\prime}$ on $z$ was neglected in the above discussions, so that the final results include only the excitation of volume plasmon. In HREM, the excitation of surface plasmon becomes important for thin specimens. Since surface excitation occurs only when the travelling electrons are very close to the surface within a distance less than about $2 \mathrm{~nm}$, it is a good approximation to consider that the plasmons of the top and bottom surfaces of the specimen (as illustrated in Fig. 1) are excited "locally" at the entrance and exit faces, respectively. For a thin slab geometry as shown in figure 1, the excitation probability of plasmons for a normal incident electron beam $[18]$ is

$$
P(\omega)=\frac{\mathrm{d} P_{\mathrm{v}}(\varepsilon)}{\mathrm{d} z} d+P_{\mathrm{s}}(\varepsilon)
$$

where $\frac{\mathrm{d} P_{\mathrm{v}}}{\mathrm{d} z}$ is the excitation probability of volume plasmon per unit distance

$$
\frac{\mathrm{d} P_{\mathrm{v}}(\varepsilon)}{\mathrm{d} z}=\frac{e^{2}}{4 \pi^{2} \varepsilon_{0} \hbar v^{2}} \operatorname{Im}\left\{-\frac{1}{\varepsilon}\right\} \ln \left[1+\left(q_{\mathrm{c}} v / \omega\right)^{2}\right]
$$

and $P_{\mathrm{s}}$ is the excitation probability of surface plasmon

$$
\begin{aligned}
P_{\mathrm{s}}(\varepsilon) & =\frac{e^{2}}{2 \pi^{2} \varepsilon_{0} \hbar v^{2}} \int_{0}^{q_{\mathrm{c}}} \mathrm{d} q \frac{q^{2}}{\left(q^{2}+(\omega / v)^{2}\right)^{2}} \times \\
& \times \operatorname{Im}\left\{-\frac{1-\varepsilon}{\varepsilon} \cdot \frac{2(\varepsilon-1) \cos (\omega d / v)+(\varepsilon-1) \exp (-q d)+\left(1-\varepsilon^{2}\right) \exp (q d)}{(\varepsilon-1)^{2} \exp (-q d)-(\varepsilon+1)^{2} \exp (q d)}\right\} .
\end{aligned}
$$




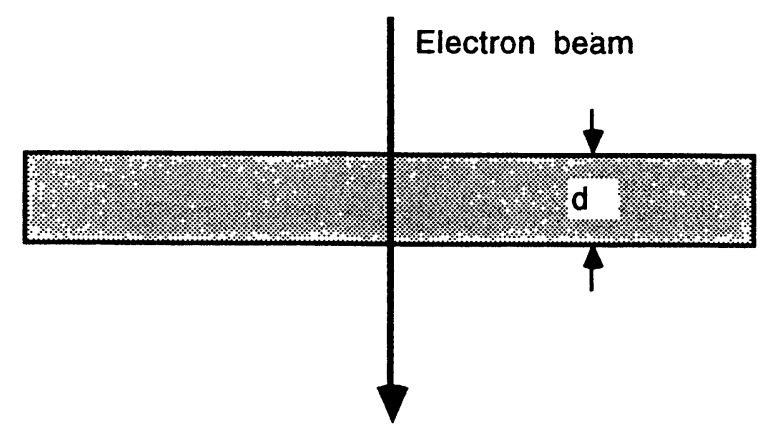

Fig. 1. - A schematic diagram showing the geometry of a thin slab foil in HREM.

Based on equation (27) and above discussions, the images formed by all the electrons can be calculated using

$$
\begin{aligned}
I(\mathbf{b}) & =\left|\Psi_{0}^{0}\left(\mathbf{b}, d, E_{0}\right) \otimes F_{\mathrm{OB}}(\mathbf{b}, 0)\right|^{2}+ \\
& +\int_{0}^{\infty} \mathrm{d} \omega \frac{\mathrm{d} P_{\mathrm{v}}(\varepsilon)}{\mathrm{d} z}\left\{k_{0}^{2}\left|Y(\mathbf{b}, \omega) \otimes F_{\mathrm{OB}}(\mathbf{b}, \omega)\right|^{2}\right\}+ \\
& +\frac{1}{2} \int_{0}^{\infty} \mathrm{d} \omega P_{\mathrm{s}}(\varepsilon)\left|\Psi_{0}^{0}\left(\mathbf{b}, d, E_{0}-\hbar \omega\right) \otimes F_{\mathrm{OB}}(\mathbf{b}, \omega)\right|^{2}+ \\
& +\frac{1}{2} \int_{0}^{\infty} \mathrm{d} \omega P_{\mathrm{s}}(\varepsilon)\left|\Psi_{0}^{0}\left(\mathbf{b}, d, E_{0}\right) \otimes F_{\mathrm{OB}}(\mathbf{b}, \omega)\right|^{2},
\end{aligned}
$$

where the first term is the image formed by purely elastically scattered electrons, the second, third and fourth terms are the contributions from the electrons after exciting the volume plasmon, the surface plasmon at the entrance and exit faces, respectively. For relatively thin crystals, equation (37) can be simplified as

$$
I(\mathbf{b}) \approx \int_{0}^{\infty} \mathrm{d} \omega\left\{\delta(\omega)+\frac{\mathrm{d} P_{\mathrm{v}}(\varepsilon)}{\mathrm{d} z} d+P_{\mathbf{s}}(\varepsilon)\right\}\left|\Psi_{0}^{0}\left(\mathbf{b}, d, E_{0}\right) \otimes F_{\mathrm{OB}}(\mathbf{b}, \omega)\right|^{2} .
$$

Figure 2 shows a comparison of simulated images of a thin GaAs [110] slab by electrons with no energy-loss $(\Delta E=0)$, volume plasmon-loss $(\Delta E=14 \mathrm{eV})$ and surface plasmon-loss $(\Delta E=$ $10 \mathrm{eV})$. The simulations were performed under the following conditions: $C_{\mathrm{s}}=1.2 \mathrm{~mm}, C_{\mathrm{c}}=$ $1.5 \mathrm{~mm}$, crystal slice thickness $0.2 \mathrm{~nm}$, specimen thickness $20 \mathrm{~nm}$, electron energy $200 \mathrm{keV}$, objective aperture radius $0.4 \AA^{-1}$, sampling point $0.109 \AA$ pixle, and energy-filter width $2 \mathrm{eV}$. The program used for the simulation was modified from Ishizuka's multislice image simulation program. The $S_{10}$ function was calculated according to equation (7) using the fast Fourier transfrom technique. At $\Delta f=43.3 \mathrm{~nm}$, there are significant differences between the images formed by elastic and volume plasmon-loss electrons; the image formed by surface-loss electrons is slightly different from that formed by elastic electrons. At Schertzer defocus, $\Delta f=63.3 \mathrm{~nm}$, the elastic image has the optimum contrast, the non-symmetric Ga-As dumbbell being identified with dark contrast. However, the inelastic images are reversed in contrast and it is not easy to distinguish the $\mathrm{Ga}$ from As atomic columns. At $\Delta f=83.3 \mathrm{~nm}$, the elastic and inelastic images are still reversal in contrast, some residual intensity appearing near the Ga columns being observed in the energyloss images. At $\Delta f=103.3 \mathrm{~nm}$, the $14 \mathrm{eV}$ energy-loss image has the optimum contrast and the 
non-symmetric Ga-As dumbbell can be identified. In the $10 \mathrm{eV}$ energy-loss image, however, the Ga-As dumbbell appears in symmetric contrast. This suggests that the inelastic scattering could complicate the image interpretation, and it is necessary to use an energy-filter for quantitative image analysis.

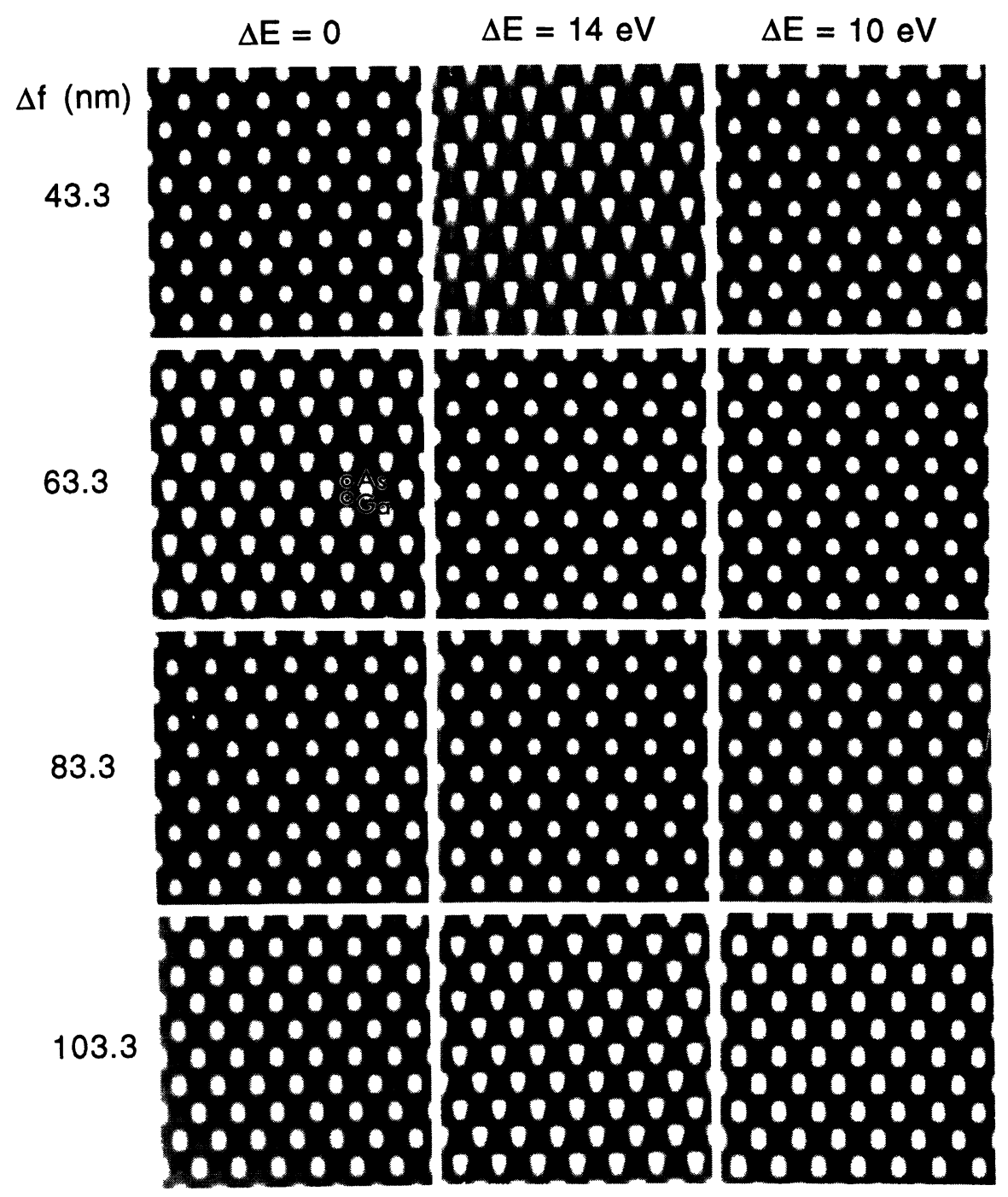

Fig. 2. - Simulated energy-filtered HREM images of a GaAs thin foil for elastic $(\Delta E=0)$, volume plasmon-loss $(\Delta E=14 \mathrm{eV})$ and surface plasmon-loss $(\Delta E=10 \mathrm{eV})$ electrons at different defocus values (see text for details). Beam azimuth is [011].

To include the angular spreading of inelastic scattering in the calculation, an incident beam of angular distribution in form of $1 /\left(\theta^{2}+\theta_{\mathrm{E}}^{2}\right)$ was used for calculating $\Psi_{0}^{0}(b, d, E)$, where the cut off value of $\theta$ was $\theta_{c}=q_{c} \lambda / 2 \pi$. The effect of large angle plasmon scattering (a few mrad) 
should be considered in this process. However, the results did not show any significant difference as compared to the calculation for parallel beam illumination case. It has been found that the dominant effect of inelastic scattering is to introduce a focus shift in the objective lens transfer function due to chromatic aberration. The calculated images using equations (37) and (38) agree very well for specimens even as thick as $40 \mathrm{~nm}$. This means that the simplified model of perfect delocalization is a good approximation in practice.

In early diffraction contrast imaging, it was illustrated by Howie [8] that the image formed by plasmon-loss electrons preserves the contrast of elastically scattered electrons. This is probably because the smaller size objective aperture used in diffraction contrast (or amplitude contrast) imaging limits the effect of chromatic aberration. However, in phase contrast HREM, the image resolution is determined by the transfer function of the objective lens, so that a small chromatic aberration would significantly affect the phase transfer properties of the lens, resulting in contrast reversal (Fig. 2). Therefore, the preservation of contrast by inelastically scattered electrons is not true in HREM.

The relative contribution of surface plasmon-loss electrons to the image is determined by the thickness of the foil. Figure 3 shows a comparison of the calculated GaAs valence-loss spectra according to equation (34) for three different foil thicknesses. The dielectric function of GaAs was obtained from the optical measurements [19]. For $d=5 \mathrm{~nm}$, the spectrum is dominated by surface excitation, the surface plasmon shifting to about $6 \mathrm{eV}$ due to the resonance coupling of the top and bottom surfaces. With the increase in the foil thickness $(d=20 \mathrm{~nm})$, the volume plasmon located at $14 \mathrm{eV}$ is stronger and the surface plasmon located at $10 \mathrm{eV}$ is relatively weak. The shift of the surface plasmon is the result of weak coupling of the two faces. For a relatively thick crystal $(d=50 \mathrm{~nm})$, the volume plasmon dominates the whole spectrum. The excitation probability of the surface plasmon reduces from 0.019 to 0.01 when the foil thickness changes from 5 to $50 \mathrm{~nm}$. The excitation probability of the volume plasmon is 0.14 for $d=50 \mathrm{~nm}$ and $E_{0}=200 \mathrm{keV}$, giving the MFP $\Lambda=357 \mathrm{~nm}$. In the thin slab case, numerical calculations have shown that the surface plasmon excitation is a relatively small factor.

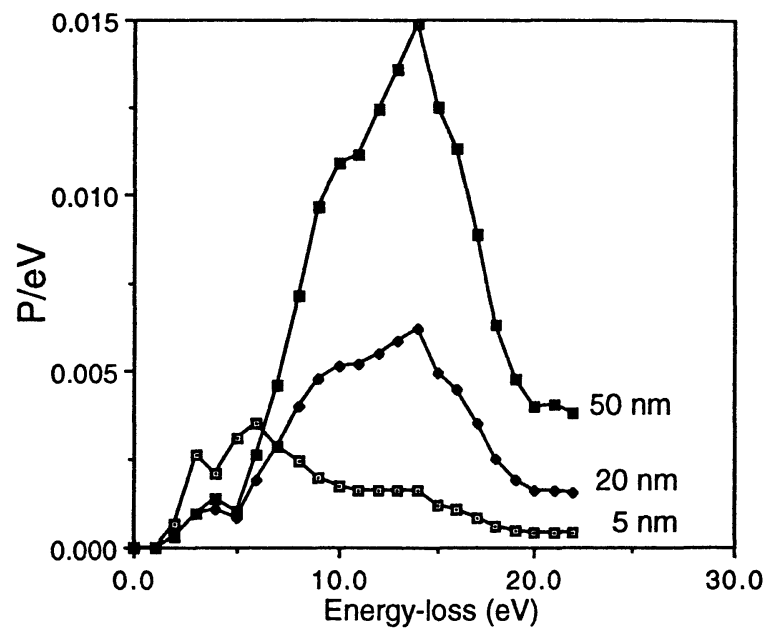

Fig. 3. - Calculated single-scattering electron energy-loss spectra for GaAs foils of thicknesses $d=5,20$ and $50 \mathrm{~nm}$. Electron energy is $200 \mathrm{keV}$, and $q_{\mathrm{c}}=1.3 \AA^{-1}$. 


\section{Energy-filtered inelastic images of interfaces.}

Further more, a more complex situation is the inelastic images of an interface of two different materials. This case happens in the surface profile and grain boundary imaging, as schematically shown in figure 4. Since the two media are characterized by different dielectric functions, $\varepsilon_{1}$ (left hand side) and $\varepsilon_{2}$ (right hand side), the excitation of the interface would depend on the distance of the electron from the interface. It is complex to consider the full dielectric resonance excitation of the entire foil. For simplification, one assumes that the excitations of the top and bottom surfaces and the interface are not strongly coupled, so that they can be treated separately. The excitation of the entrance and exit surfaces can be approximately described by equation (34). By neglecting the finite size of the slab, the interface excitation could be treated using Howie's method for an electron moving parallel to a large plane [20]. The image formed by all the transmitted electrons may be written as

$$
\begin{aligned}
& I(\mathbf{b})=\left|\Psi_{0}^{0}\left(\mathbf{b}, d, E_{0}\right) \otimes F_{\mathrm{OB}}(\mathbf{b}, 0)\right|^{2}+ \\
& +\int_{0}^{\infty} \mathrm{d} \omega\left\{\frac{\mathrm{d} P_{\mathrm{v}}\left(\varepsilon_{1}, x\right)}{\mathrm{d} z} \theta(-x)+\frac{\mathrm{d} P_{\mathrm{v}}\left(\varepsilon_{2}, x\right)}{\mathrm{d} z} \theta(x)\right\}\left\{k_{0}^{2}\left|Y(\mathbf{b}, \omega) \otimes F_{\mathrm{OB}}(\mathbf{b}, \omega)\right|^{2}\right\}+ \\
& +\frac{1}{2} \int_{0}^{\infty} \mathrm{d} \omega\left\{P_{\mathrm{s}}\left(\varepsilon_{1}\right) \theta(-x)+P_{\mathrm{s}}\left(\varepsilon_{2}\right) \theta(x)\right\}\left|\Psi_{0}^{0}\left(\mathbf{b}, d, E_{0}-\hbar \omega\right) \otimes F_{\mathrm{OB}}(\mathbf{b}, \omega)\right|^{2}+ \\
& +\frac{1}{2} \int_{0}^{\infty} \mathrm{d} \omega\left\{P_{\mathrm{s}}\left(\varepsilon_{1}\right) \theta(-x)+P_{\mathrm{s}}\left(\varepsilon_{2}\right) \theta(x)\right\}\left|\Psi_{0}^{0}\left(\mathbf{b}, d, E_{0}\right) \otimes F_{\mathrm{OB}}(\mathbf{b}, \omega)\right|^{2}+ \\
& +\int_{0}^{\infty} \mathrm{d} \omega\left\{\frac{\mathrm{d} P_{\mathrm{i}}\left(\varepsilon_{1}, \varepsilon_{2}, x\right)}{\mathrm{d} z} \theta(-x)+\frac{\mathrm{d} P_{\mathrm{i}}\left(\varepsilon_{2}, \varepsilon_{1}, x\right)}{\mathrm{d} z} \theta(x)\right\}\left\{k_{0}^{2}\left|Y(\mathbf{b}, \omega) \otimes F_{\mathrm{OB}}(\mathbf{b}, \omega)\right|^{2}\right\},
\end{aligned}
$$

where the first four terms have a similar meaning as those defined for equation (37), the fifth term is the result of interface excitation

$$
\frac{\mathrm{d} P_{\mathrm{i}}\left(\varepsilon_{1}, \varepsilon_{2}, x\right)}{\mathrm{d} z}=\frac{e^{2}}{2 \pi^{2} \varepsilon_{0} \hbar v^{2}} \operatorname{Im}\left\{\frac{2}{\varepsilon_{1}+\varepsilon_{2}}\right\} K(2 \omega x / v) ;
$$

the volume plasmon excitation probability depends on the distance of the electrons from the interface

$$
\begin{gathered}
\frac{\mathrm{d} P_{\mathrm{v}}(\varepsilon, x)}{\mathrm{d} z}=\frac{e^{2}}{2 \pi^{2} \varepsilon_{0} \hbar v^{2}} \operatorname{Im}\left\{-\frac{1}{\varepsilon}\right\}\left[\ln \left(q_{\mathrm{c}} v / \omega\right)-K(2 \omega x / v)\right] \\
K(2 \omega x / v)=\int_{0}^{q_{\mathrm{c}}} \mathrm{d} q \frac{\exp \left[-2 x\left(q^{2}+\omega^{2} / v^{2}\right)^{1 / 2}\right]}{\left(q^{2}+\omega^{2} / v^{2}\right)^{1 / 2}}
\end{gathered}
$$

and $\theta(x)$ is a step function

$$
\theta(x)=\left\{\begin{array}{l}
1 \text { for } x>0 \\
0 \text { for } x<0
\end{array}\right.
$$

For thin crystals, equation (39) can be simplified as

$$
\begin{aligned}
I(\mathbf{b}) \approx & \int_{0}^{\infty} \mathrm{d} \omega\left\{\delta(\omega)+\left[\frac{\mathrm{d} P_{\mathrm{v}}\left(\varepsilon_{1}, x\right)}{\mathrm{d} z} d+P_{\mathrm{s}}\left(\varepsilon_{1}\right)\right] \theta(-x)+\right. \\
& \left.+\left[\frac{\mathrm{d} P_{\mathrm{v}}\left(\varepsilon_{2}, x\right)}{\mathrm{d} z} d+P_{\mathrm{s}}\left(\varepsilon_{2}\right)\right] \theta(x)+\frac{\mathrm{d} P_{\mathrm{i}}\left(\varepsilon_{2}, \varepsilon_{1}, x\right)}{\mathrm{d} z} d\right\}\left|\Psi_{0}^{0}\left(\mathbf{b}, d, E_{0}\right) \otimes F_{\mathrm{OB}}(\mathbf{b}, \omega)\right|^{2}
\end{aligned}
$$




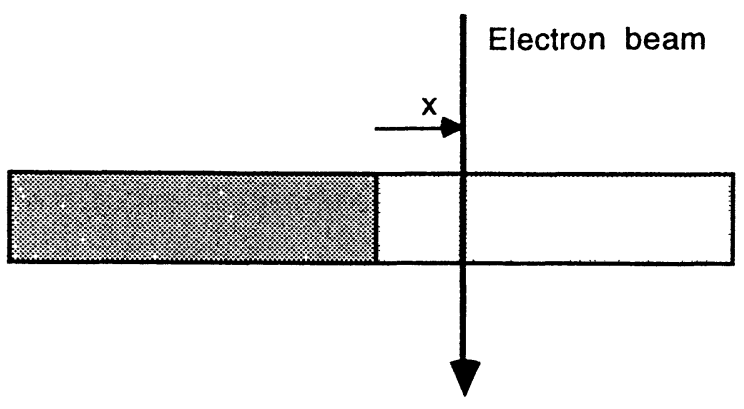

Fig. 4. - A schematic diagram showing the geometry of an interface between two adjacent thin foils. $x$ indicates the position of the incident electrons with respect to the interface.

where the images formed by the elastic, volume and surface plasmon-loss, and interface-loss electrons are characterized by the $\delta, \theta$ and $\frac{\mathrm{d} P_{\mathrm{i}}}{\mathrm{d} z}$ terms, respectively. It is important to note that the excitations of volume $\left(P_{\mathrm{v}}\right)$ and interface $\left(P_{\mathrm{i}}\right)$ plasmons depend on the relative position of the electrons with respect to the interface. It is this effect that will introduce some additional contrast in the inelastic images of interfaces, as described below.

To simplify the discussion, the surface profile imaging of GaAs (100) can be taken as an example. Figure 5 shows the calculated excitation probabilities of volume and surface plasmons for a $50 \mathrm{~nm}$ thick foil. The variations of $\mathrm{d} P_{\mathrm{v}} / \mathrm{d} z$ and $\mathrm{d} P_{\mathrm{i}} / \mathrm{d} z$ are quite slow when the electrons move close to the interface from the GaAs side $(x<0)$. The only sharp change occurs when the electron is about $0.3 \mathrm{~nm}$ away from the interface. In the vacuum side $(x>0)$, the interaction of the electron beam with the interface is a long range interaction, resulting in some residual surface plasmon excitation even at $x>3 \mathrm{~nm}$. This is probably the nature of non-localized inelastic excitation. In the vacuum side, only surface excitation would occur. Although the variations of $\mathrm{d} P_{\mathrm{v}} / \mathrm{d} z$ and $\mathrm{d} P_{\mathrm{i}} / \mathrm{d} z$ are quite significant near the interface, the total excitation probability $\mathrm{d} P / \mathrm{d} z=\mathrm{d} P_{\mathrm{i}} / \mathrm{d} z+$ $\mathrm{d} P_{\mathrm{v}} / \mathrm{d} z$ is almost a constant inside GaAs. Figure 6 shows a comparison of the calculated spectra for electrons travelling at different distances from the interface. At the interface $(x=0)$, the surface plasmon at $10 \mathrm{eV}$ is strongly excited. When the electrons move further away into the foil $(x=-0.5 \mathrm{~nm})$, the volume plasmon is excited. At $x=-2 \mathrm{~nm}$, the whole spectrum is dominated by volume plasmon excitation.

Surface plasmon excitation is important in dynamic calculations for reflection electron microscopy [21], because the incident electrons would be trapped by the surface for a distance about $100 \mathrm{~nm}$ before being reflected into the vacuum. This situation also happens for surface profile imaging, in which the electrons travelling near the surface would suffer more surface-losses, as illustrated in figure 5. This localization effect would affect the image contrast. Figure 7 shows the simulated surface profile images of $\mathrm{GaAs}(100)$ viewed along [011] for different energy-losses. For simplification, the GaAs (100) is assumed as a perfect surface without atomic reconstruction. For the elastically scattered electrons, besides the regular lattice images in the GaAs side, some residual contrast is also observed in the vacuum side. For the inelastic images, due to the variations of excitation probabilities close to the surface (or interface), as shown in figure 5 , the decrease and increase of image contrast for 14 (volume plasmon) and $10 \mathrm{eV}$ (surface plasmon) losses, respectively, are significant. This may be important in image interpretation. It is worth pointing out that the intensity of energy-filtered surface-loss image does not go to zero at distances far away from the surface, because the surface and volume plasmon peaks for GaAs are not sharp enough so 


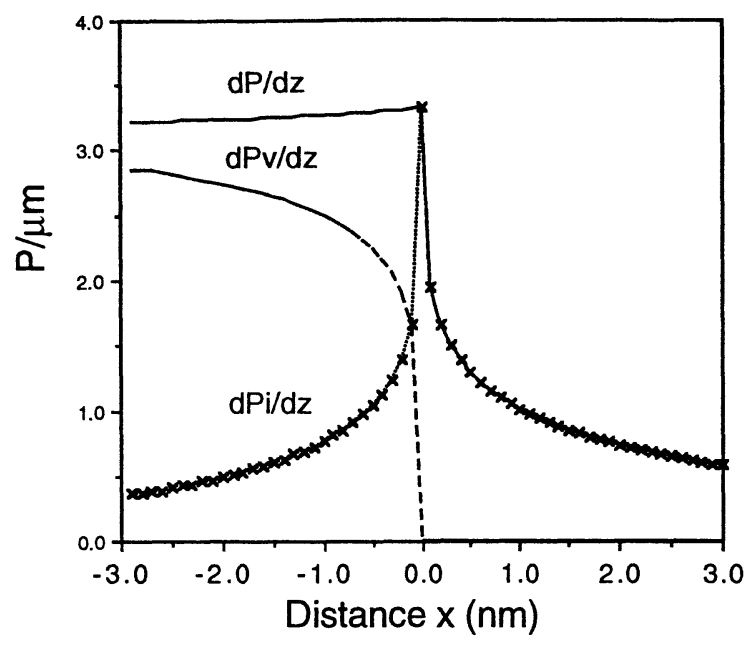

Fig. 5. - Calculated GaAs volume plasmon $\left(\mathrm{d} P_{\mathrm{v}} / \mathrm{d} z\right)$ and interface plasmon (i.e., GaAs with vacuum) $\left(\mathrm{d} P_{\mathrm{i}} / \mathrm{d} z\right)$ excitation probabilities per unit distance as a function of the impact parameter $x . \mathrm{d} P / \mathrm{d} z=\mathrm{d} P_{\mathrm{v}} / \mathrm{d} z+$ $\mathrm{d} P_{\mathrm{i}} / \mathrm{d} z$ is the total excitation probability of plasmons, with $x<0$ inside the crystal and $x>0$ in the vacuum. The variation of $\mathrm{d} P_{\mathrm{i}} / \mathrm{d} z$ close to the interface $(x=0)$ indicates the localization effect of surface excitations. Electron energy is $200 \mathrm{keV}$.

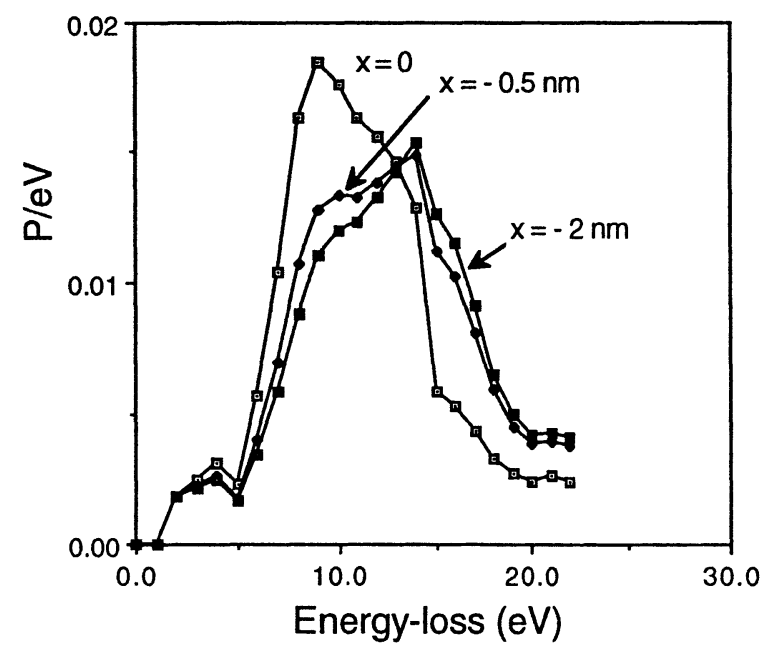

Fig. 6. - Calculated single-scattering electron energy-loss spectra for the GaAs-vacuum interface for impact parameters $x=0,-0.5$ and $-2 \mathrm{~nm}$. The foil thickness is $50 \mathrm{~nm}$, and electron energy is $200 \mathrm{keV}$.

that the finite width of the energy-filter would receive the contribution from the tail part of the volume plasmon.

In addition, a sharp line contrast is observed at the surface in the $10 \mathrm{eV}$ loss images in figure 7. This effect is an artifact produced in following ways. In classical dielectric theory, a surface is treated as a plane without thickness, and the surface charges are assumed to distribute in this plane. This is actually not precise at the atomic level. Ignoring the dispersion dependence of 
$\varepsilon(\omega)$ actually sets the values of the $K$ function (Eq. (42)) very high at $x=0$, resulting in a sharp intensity wall at $x=0$. This artifact can be removed by using more rigorous dielectric theory [22].

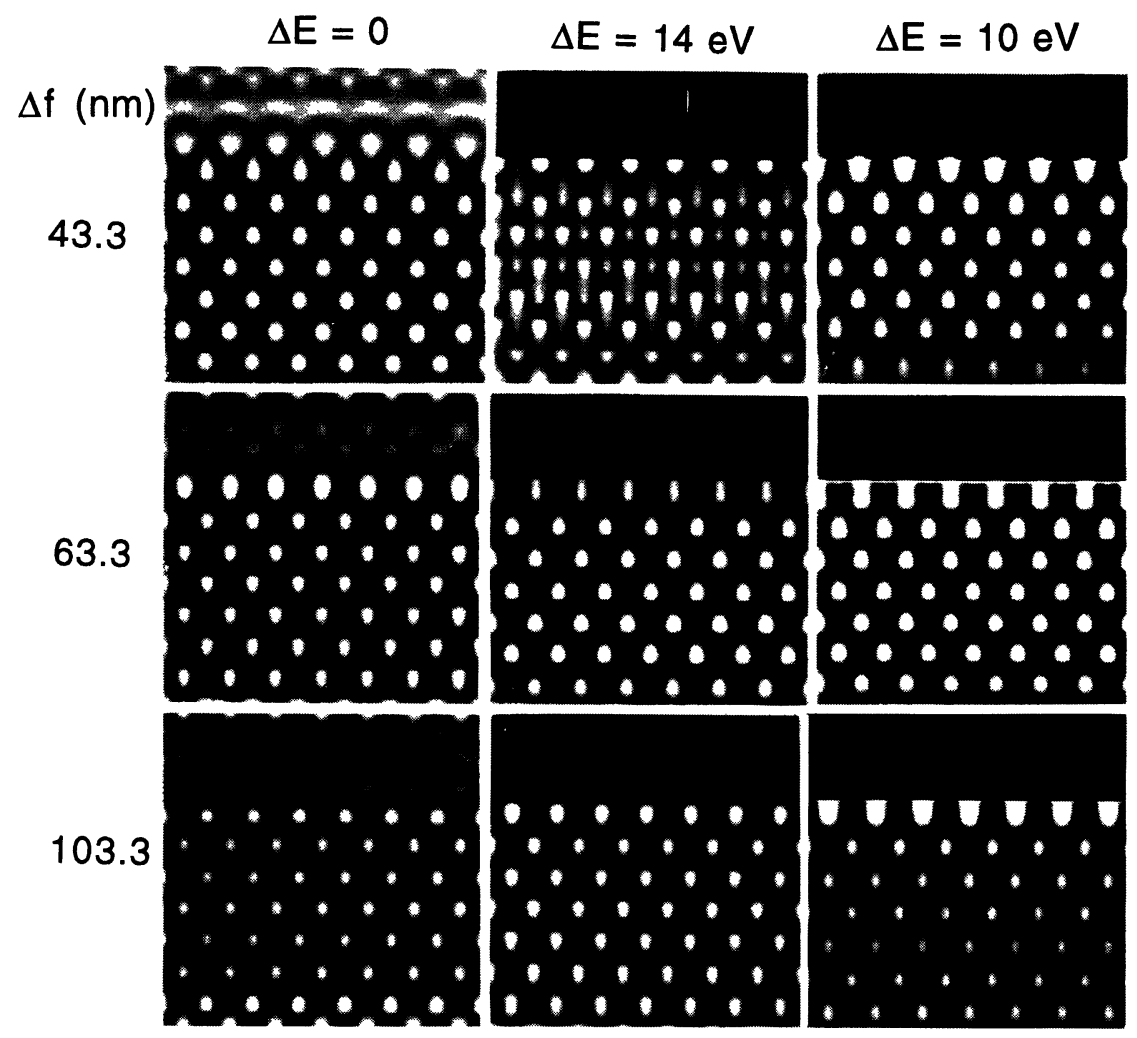

Fig. 7. - Simulated energy-filtered HREM surface profile images of GaAs (100) of elastic $(\Delta E=0)$, volume plasmon-loss $(\Delta E=14 \mathrm{eV})$ and surface plasmon-loss $(\Delta E=10 \mathrm{eV})$ electrons. The simulation conditions are the same as those for figure 2. Beam azimuth is [011].

Following the theoretical scheme discussed above, the excitation of interfaces in finite semiconductor superlattices can also be included in image simulation by using the corresponding excitation probability function [23].

Besides the contribution of valence excitation, phonon or thermal diffuse scattering is another important inelastic process in HREM imaging. Localized phonon excitation can scatter the electrons into the angular range outside the objective aperture. This is equivalent to introducing an absorption potential in the calculation of elastic wave. This absorption factor can be calculated either in real $[24,25]$ or reciprocal $[26$ space. The phonon scattered electrons falling inside the objective aperture angular range form incoherent atomic resolution image. Calculation of this type of image can also be performed using equation (5), as described elsewhere [9].

\section{Conclusion.}

In conclusion, wave mechanics has been applied to treat inelastic valence excitation in high-energy 
electron diffraction and imaging. A general theory has been presented to calculate the diffraction patterns and HREM images of the valence excited electrons based on single inelastic scattering model. The incoherence of the different valence states has been evaluated before any numerical calculations. This new theory reduces to the simplified theory proposed by several authors [1-3] under the small thickness approximation.

For a specimen thinner than the localization of the valence excitations, the valence-loss can be treated as occurring at the entrance face of the crystal foil; their propagation through the crystal can be considered as the elastic scattering of incident inelastic electrons. The inelastic scattering diffraction pattern is formed by the incoherent sum of the "elastically" scattered electrons with different incident energies, being weighted by the intensity distribution in the electron energy-loss spectrum. A similar method can be applied to consider the inelastic effect in HREM images. In addition, a convolution of the objective lens transfer function for electrons with different energies is required.

The proposed theory has been generalized to include the surface and interface excitation in HREM image simulations for surfaces and grain boundary interfaces. Calculations have shown that the localization effect of surface plasmon excitation in profile imaging may introduce some contrast variation near the surface.

\section{Acknowledgements.}

This research was sponsored by the Division of Materials Sciences, U.S. Department of Energy, under contract DE-AC05-84OR21400 with Martin Marietta Energy Systems, Inc.

\section{Appendix A}

Equivalence of equation (5) with the inelastic multislice theory.

For easy notation, one separates the wave function from its plane wave part by defining

$$
\begin{aligned}
& \Psi_{n}(\mathbf{r})=\varphi_{n}(\mathbf{b}, z) \exp \left(i \mathbf{k}_{n} \cdot \mathbf{r}\right) \\
& \Psi_{n}^{0}(\mathbf{r})=\varphi_{n}^{0}(\mathbf{b}, z) \exp \left(i \mathbf{k}_{n} \cdot \mathbf{r}\right)
\end{aligned}
$$

Thus equation (5b) can be rewritten as

$$
\varphi_{n}(\mathbf{b}, z)=\alpha \int_{0}^{z} \mathrm{~d} z^{\prime}\left\{H_{n 0}^{\prime}\left(\mathbf{b}, z^{\prime}\right) \Psi_{0}^{0}\left(\mathbf{b}, z^{\prime}\right) / \frac{\partial \Psi_{n}^{0}\left(\mathbf{b}, z^{\prime}\right)}{\partial z}\right\} \varphi_{n}^{0}(\mathbf{b}, z),
$$

By neglecting the back scattering term under the small angle approximation, in the multislice method, the solution of the elastic Schrödinger equation can be written as $[5,10]$

$$
\varphi_{n}^{0}(\mathbf{b}, z+\Delta z)=\left(\varphi_{n}^{0}(\mathbf{b}, z) Q_{0}(\mathbf{b})\right) \otimes P_{n}(\mathbf{b}) .
$$

where $P_{n}$ is the propagation function of the wave in the vacuum for a distance $\Delta z=z-z_{0}$,

$$
P_{n}(\mathbf{b}) \equiv \frac{1}{i \Delta z \lambda_{n}} \exp \left(i \pi b^{2} / 2 \lambda_{n} \Delta z\right)
$$

$\sigma=\frac{1}{\hbar v} ;$ and $\lambda_{n}$ is the electron wavelength of energy $E_{n}$, and $Q_{0}$ is the so-called phase grating function of the slice,

$$
Q_{0}(\mathbf{b}) \equiv \exp \left[i e \sigma \int_{z}^{z+\Delta z} \mathrm{~d} z V(\mathbf{b}, z)\right]
$$


Now one derives the relationship which governs the inelastic wave $\varphi_{n}(\mathbf{b}, z)$ before and after being scattered by a very thin crystal slice of thickness $\Delta z$. From equation (A2), one can directly write

$$
\begin{aligned}
& \varphi_{n}(\mathbf{b}, z+\Delta z)-\varphi_{n}(\mathbf{b}, z)=\alpha \int_{0}^{z} \mathrm{~d} z^{\prime}\left\{H_{n 0}^{\prime}\left(\mathbf{b}, z^{\prime}\right) \Psi_{0}^{0}\left(\mathbf{b}, z^{\prime}\right) / \frac{\partial \Psi_{n}^{0}\left(\mathbf{b}, z^{\prime}\right)}{\partial z}\right\} \times \\
& \times\left[\varphi_{n}^{0}(\mathbf{b}, z+\Delta z)-\varphi_{n}^{0}(\mathbf{b}, z)\right]+ \\
& +\alpha \int_{z}^{z+\Delta z} \mathrm{~d} z^{\prime}\left\{H_{n 0}^{\prime}\left(\mathbf{b}, z^{\prime}\right) \Psi_{0}^{0}\left(\mathbf{b}, z^{\prime}\right) / \frac{\partial \Psi_{n}^{0}\left(\mathbf{b}, z^{\prime}\right)}{\partial z}\right\} \varphi_{n}^{0}(\mathbf{b}, z+\Delta z) \\
& \approx \varphi_{n}(\mathbf{b}, z)\left[\frac{\varphi_{n}^{0}(\mathbf{b}, z+\Delta z)}{\varphi_{n}^{0}(\mathbf{b}, z)}-1\right]+\alpha\left\{h_{n 0}^{\prime}(\mathbf{b}, z) \Psi_{0}^{0}(\mathbf{b}, z) / \frac{\partial \Psi_{n}^{0}(\mathbf{b}, z)}{\partial z}\right\} \varphi_{n}^{0}(\mathbf{b}, z+\Delta z) .(A
\end{aligned}
$$

where $h_{n 0}^{\prime}(\mathbf{b}, z)=\int_{z}^{z+\Delta z} \mathrm{~d} z H_{n 0}^{\prime}(\mathbf{b}, z)$. Equation (A6) can be conveniently written as

$\varphi_{n}(\mathbf{b}, z+\Delta z) \approx \varphi_{n}(\mathbf{b}, z)\left(\frac{\varphi_{n}^{0}(\mathbf{b}, z+\Delta z)}{\varphi_{n}^{0}(\mathbf{b}, z)}\right)+\alpha\left\{h_{n 0}^{\prime}(\mathbf{b}, z) \Psi_{0}^{0}(\mathbf{b}, z) / \frac{\partial \Psi_{n}^{0}(\mathbf{b}, z)}{\partial z}\right\} \varphi_{n}^{0}(\mathbf{b}, z+\Delta z)$.

For fast electrons, it is always a good approximation to assume forward scattering, so that

$$
\frac{\partial \Psi_{n}^{0}(\mathbf{b}, z)}{\partial z}=\left[i k_{n z} \varphi_{n}^{0}(\mathbf{b}, z)+\frac{\partial \varphi_{n}^{0}(\mathbf{b}, z)}{\partial z}\right] \exp \left(i \mathbf{k}_{n} \cdot \mathbf{r}\right) \approx i k_{n z} \varphi_{n}^{0}(\mathbf{b}, z) \exp \left(i \mathbf{k}_{n} \cdot \mathbf{r}\right)
$$

Using equation (A8), equation (A7) becomes

$$
\varphi_{n}(\mathbf{b}, z+\Delta z) \approx\left[\varphi_{n}(\mathbf{b}, z)-i \sigma h_{n 0}^{\prime}(\mathbf{b}, z) \varphi_{0}^{0}(\mathbf{b}, z)\right]\left(\frac{\varphi_{n}^{0}(\mathbf{b}, z+\Delta z)}{\varphi_{n}^{0}(\mathbf{b}, z)}\right)
$$

The physical meaning of equation (A9) can be interpreted as follows. The first term in [...] $\left(\varphi_{n}(\mathbf{b}, z)\right)$ is the inelastic wave generated before the wave arrives the slice entrance face located at $z=z$. In the single inelastic scattering model, this part of the wave will only be elastically scattered when penetrating the crystal slice and is responsible for the formation of Kikuchi patterns. The second term in [...] $\left(\sigma h_{n 0}^{\prime} \varphi_{0}\right)$ is the newly generated inelastic wave when the elastic wave $\left(\varphi_{0}^{0}\right)$ penetrates through the slice. The elastic scattering of these two parts within the slice is included in the term $\varphi_{n}^{0}(\mathbf{b}, z+\Delta z) / \varphi_{n}^{0}(\mathbf{b}, z)$. For a very thin slice $(\Delta z \rightarrow 0), P_{n}$ is very close to a Dirac $\delta$ function. Using equation (A3), equation (A9) can thus be approximately written as

$$
\begin{aligned}
\varphi_{n}(\mathbf{b}, z+\Delta z) & =\left[\varphi_{n}(\mathbf{b}, z)-i \sigma h_{n 0}^{\prime}(\mathbf{b}, z) \varphi_{0}^{0}(\mathbf{b}, z)\right] \frac{\left(\varphi_{n}^{0}(\mathbf{b}, z) Q_{0}(\mathbf{b}, z)\right) \otimes P_{n}(\mathbf{b})}{\varphi_{n}^{0}(\mathbf{b}, z)} \\
& \approx\left[Q_{0}(\mathbf{b}, z)\left\{\varphi_{n}(\mathbf{b}, z)-i \sigma h_{n 0}^{\prime}(\mathbf{b}, z) \varphi_{0}^{0}(\mathbf{b}, z)\right\}\right] \otimes P_{n}(\mathbf{b}) .
\end{aligned}
$$

Equation (A10) is the exact form of the inelastic multislice theory for single inelastic scattering $[6,11-12]$. 


\section{Appendix B}

Perturbation theory for calculating $\Psi_{0}^{0}\left(\mathbf{r}, E_{0}-\Delta E\right)$ from $\Psi_{0}^{0}\left(\mathbf{r}, E_{0}\right)$.

$\Psi_{0}^{0}(\mathbf{r}, E-\Delta E)$ and $\Psi_{0}^{0}(\mathbf{r}, E)$ are determined by equations

$$
\left(\nabla^{2}+\frac{2 m_{0}}{\hbar^{2}}\left(E_{0}-\Delta E\right)\right) \Psi_{0}^{0}(\mathbf{r}, E-\Delta E)=-\frac{2 m_{0} e}{\hbar^{2}} V(\mathbf{r}) \Psi_{0}^{0}\left(\mathbf{r}, E_{0}-\Delta E\right)
$$

and

$$
\left(\nabla^{2}+\frac{2 m_{0}}{\hbar^{2}} E_{0}\right) \Psi_{0}^{0}\left(\mathbf{r}, E_{0}\right)=-\frac{2 m_{0} e}{\hbar^{2}} V(\mathbf{r}) \Psi_{0}^{0}\left(\mathbf{r}, E_{0}\right)
$$

If $\Psi_{0}^{0}(\mathbf{r}, E-\Delta E)$ can be written in a form

$$
\Psi_{0}^{0}\left(\mathbf{r}, E_{0}-\Delta E\right)=\rho(\mathbf{r}) \Psi_{0}^{0}\left(\mathbf{r}, E_{0}\right),
$$

for $\Delta E<<E_{0}$, it is believed that $\rho(\mathbf{r})$ is a slow varying function. By neglecting the $\nabla^{2} \rho$ term, for $k_{x, y}<<k_{z}$, so that the $\partial \Psi_{0}^{0}(\mathbf{r}) / \partial x$ and $\partial \Psi_{0}^{0}(\mathbf{r}) / \partial y$ terms are relatively small, the equation satisfied by $\rho(\mathbf{r})$ is

$$
\frac{\partial \rho(\mathbf{r})}{\partial z} \approx \frac{m_{0}}{\hbar^{2}} \Delta E \Psi_{0}^{0}\left(\mathbf{r}, E_{0}\right)\left[\partial \Psi_{0}^{0}\left(\mathbf{r}, E_{0}\right) / \partial z\right]^{-1} \rho(\mathbf{r})
$$

Solving equation (B4) and get

$$
\rho(\mathbf{r})=\exp \left\{k^{2} \theta_{\mathrm{E}} \int_{0}^{z} \mathrm{~d} z^{\prime} \Psi_{0}^{0}\left(\mathbf{b}, z^{\prime}, E_{0}\right)\left[\partial \Psi_{0}^{0}\left(\mathbf{b}, z^{\prime}, E_{0}\right) / \partial z\right]^{-1}\right\} .
$$

Therefore

$$
\Psi_{0}^{0}\left(\mathbf{r}, E_{0}-\Delta E\right) \approx \exp \left\{k^{2} \theta_{\mathrm{E}} \int_{0}^{z} \mathrm{~d} z^{\prime} \Psi_{0}^{0}\left(\mathbf{b}, z^{\prime}, E_{0}\right)\left[\partial \Psi_{0}^{0}\left(\mathbf{b}, z^{\prime}, E_{0}\right) / \partial z\right]^{-1}\right\} \Psi_{0}^{0}\left(\mathbf{r}, E_{0}\right)
$$

Numerical calculations showed that $\Psi_{0}^{0}\left(\mathbf{r}, E_{0}-\Delta E\right) / \Psi_{0}^{0}\left(\mathbf{r}, E_{0}\right)$ is very close to 1 even for a $40 \mathrm{~nm}$ thick foil.

\section{References}

[1] STOBBs W.M. and SAXTON W.O., J. Microsc. 151 (1988) 171;

BOOTHROYD C.B. and STOBBS W.M., Ultramicroscopy 31 (1989) 259.

[2] Tang D., Brydson R.D., JefFerson D.A. and Thomas J.M., J. Phys.: Cond. Matter 1 (1989) 1561.

[3] KRIVANEK O.L., AHN C.C. and WOOD G.J., Ultramicroscopy 33 (1990) 177.

[4] COWLEY J.M. and MOODIE A.F., Acta. Cryst. A10 (1957) 609.

[5] Yoshioka H., J. Phys. Soc. J. 12 (1957) 618.

[6] WANG Z.L., Acta Cryst. A45 (1989) 636;

WANG Z.L., Phys. Rev. B41 (1990) 12818.

[7] WANG Z.L. and BENTLEY J., Ultramicroscopy 38 (1991) 181.

[8] HowI A., Proc. Roy. Soc. London 271 (1963) 268.

[9] WANG Z.L., Philos. Mag. (1992) in press.

[10] ISHIZUKA K. and UYEDA N., Acta Cryst. A33 (1977) 740. 
[11] Cowley J.M. and Pagany A.P., Acta Cryst. A24 (1968) 109.

[12] DOYLE P.A., Acta Cryst. A25 (1969) 569;

DOYLE P.A., Acta Cryst. A27 (1971) 109.

[13] KOHL H. and ROSE H., Adv. Electron. Electron Phys. 65 (1985) 173.

[14] ADLER S., Phys. Rev. 126 (1962) 413.

[15] RAETHER H., Excitation of plasmons and interband transitions by electrons, Springer Tracts Mod. Phys. Vol. 88 (Springer-Verlag, New York, 1980).

[16] GJøNNES G. and HøIER R., Acta Cryst. A27 (1971) 166.

[17] PENNYCOOK S.J. and HOWIES A., Philos. Mag. A41 (1980) 809.

[18] RitCHIE R.H., Phys. Rev. 106 (1957) 874.

[19] Handbook of optical constants of solids, E.D. Palik Ed. (Academic Press, Inc. New York, 1985).

[20] HowIE A., Ultramicroscopy 11 (1983) 141.

[21] WANG Z.L., Surf. Sci. 215 (1989) 217.

[22] ZABALA N. and ECHENIQUE P.M., Ultramicroscopy 32 (1990) 327.

[23] NKOMA J.S., Surf. Sci 191 (1987) 595.

[24] WANG Z.L. and BeNTLEY J., Inst. Phys. Conf. Proc. Ser. No. 119 (1991) 547.

[25] WANG Z.L. and COWLEY J.M., Ultramicroscopy 32 (1990) 275.

[26] BIRD D.M. and KING Q.A., Acta Cryst. A46 (1990) 202. 